\title{
Preliminary results on the Multipactor effect prediction in RF components with ferrites
}

\author{
V.E. Semenov, E. Rakova, M. Belhaj, J. Puech, M. Lisak, J. Rasch, E. Laroche \\ CNES, 18 Avenue Edouard Belin 31401 Toulouse Cédex 9, France \\ Institute of Applied Physics RAS, 46 Ulyanov St. 603950 Nizhny Novgorod, Russia \\ ONERA/DESP, BP4025, 2 Av. Edouard Belin, 31055 Toulouse Cédex 4, FRANCE \\ Chalmers University of Technology SE - 41296 Göteborg, Sweden \\ COBHAM, 31 Av. De la baltique - ZA de Courtaboeuf, 91978 Villebon-sur-Yvette Cédex, France
}

\begin{abstract}
This paper deals with the analysis of the Multipactor effect in RF components with ferrites performed through the preliminary measurements of ferrites secondary emission coefficient and the simulations of the effect of a continuous magnetic field on the electron bunch spread. The impact of this effect on the Multipactor threshold was quantified.
\end{abstract}

Keywords: Multipactor, isolators, circulators, ferrites, secondary emission coefficient.

\section{Introduction}

Multipactor breakdown is an important potential failure mechanism in many different microwave devices working under close to vacuum conditions. It can be described as an avalanche-like phenomenon resulting from the collision of primary electrons with the component wall creating thus the emission of secondary electrons. Under certain conditions of dimensions, material, rf electromagnetic field characteristics, the electron density can grow exponentially leading to the discharge. Applications range from space borne $\mathrm{rf}$ equipment to high-power microwave generators and charged particle accelerators. The basic physics involved in the multipactor phenomenon is well known for the case of a homogeneous rf field between two infinite parallel plates. However, most realistic rf device such as circulators and isolators involve inhomogeneous rf electric fields, complex material such as ferrites and the use of permanent magnetic field [1] [2]. In such situations, the evaluation of the Multipactor risks can be complicated as no prediction means were set-up.

In a first part, some preliminary measurements of the ferrites secondary emission coefficient versus the incident electrons energy will allow to confirm the complexity of the Multipactor analysis with complex material. Then the second part will deal with the Multipactor analysis within the gap between the ferrite disks with a permanent magnetic field.

\section{Ferrites secondary emission coefficient measurements}

Ferrites are dielectric materials. Consequently, their electrical behavior under irradiation has to be determined. As a matter of fact, the accumulated electric charge can modify the wall - electron interaction. Without any leakage current, compensation phenomena lead to an equilibrium state corresponding to the equality between the sample input and output current. In that context, the classical method based on current measurement [3] cannot be applied and the KP probe method must be used. Two kinds of ferrite samples were measured with the classical current method.

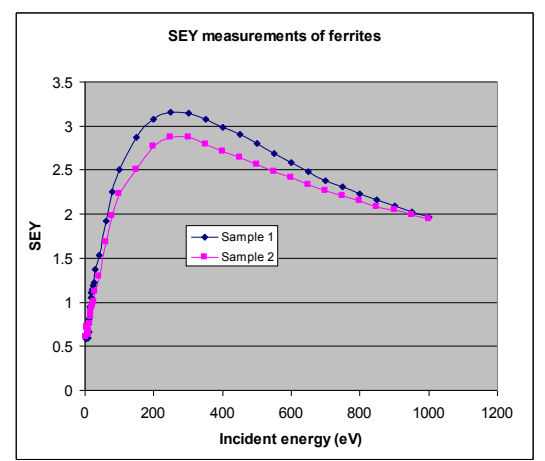

Figure 1. SEY measurements of 2 ferrite samples

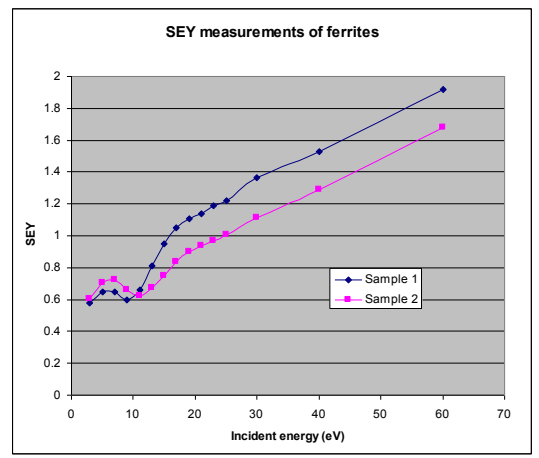

Figure 2. SEY measurements at very low energy The samples are characterized by high secondary emission coefficient. It can be explained by the fact that these samples have a dielectric-like behavior : the electrons electrons interaction are rare compared to the case of metal, what enables generated electrons inside the metal to reach the surface more easily.

\section{Effect of the permanent magnetic field on the Multipactor threshold}

The main difficulty in the analysis of the Multipactor effect in RF components containing ferrite lies on the fact that this material is a gyrotropic medium controlled by an applied permanent magnetic field. A simplified scheme of such a component can be presented as follows : 


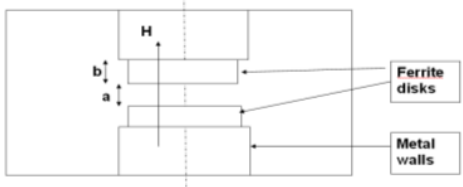

A Particle-In-Cell code, similar to the one developed in [4] was developed to demonstrate the influence of the permanent magnetic field with 2 different values of gap width. The simulations were performed using Vaughan's approximation [5] and the value of the first cross-over point at around $30 \mathrm{eV}$ like the sample 2 of the previous paragraph. The electric field cartography between the 2 disks is such that the axial component of this field is constant along the height and that the transverse components vary smoothly. In all following graphs, the dashed red line corresponds to localized seed electrons, the solid red line corresponds to uniformly distributed seed electrons, the black line corresponds to uniform rf electric field and the blue line corresponds to uniform rf field with averaging over the disk surface.

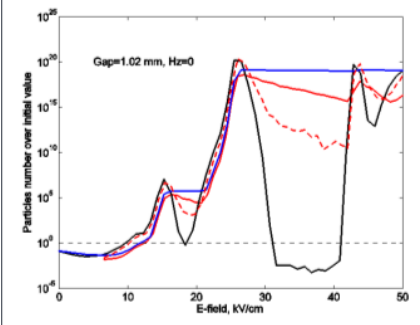

(a)

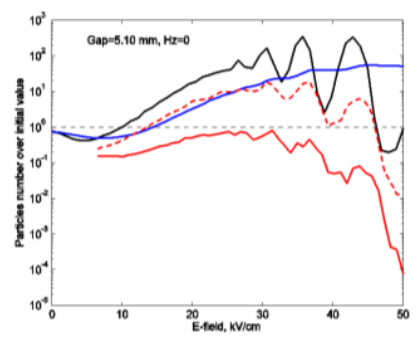

(c)

Figure 3.a, b, c, d : Relative increase of the number of electrons after 100 if periods versus the if field amplitude

From the general point of view, the introduction of a permanent magnetic field does not influence the determination of the threshold in the case of plane parallel model analysis. In the case of our component, we notice that localized seed electrons give the same threshold values as in the case of the simple plane parallel model. It means that the magnetic field prevents the electrons from drifting outside the region between the 2 ferrite disks (dashed red and solid black curves of fig. 3.a and b). The results with uniformly distributed seed electrons are similar to the ones obtained by averaging within the plane parallel model (solid red and blue lines of fig. 3.a and b). In the case of a wider gap, the resonant Multipactor zones are narrower due to the spread of the electron initial velocities. Without magnetic field in the 3D simulations with uniformly distributed seed electrons, the number of electrons decreases with increased RF field amplitude, due to the drift of electrons out of the critical region (Miller force) [6]. The figure 3.d shows that the permanent magnetic field stops the electrons drift, even if we are in the case of a wide gap.

\section{Conclusions and perspectives}

In the case of a configuration with 2 ferrite disks, the fringing field phenomena due to the Miller force and to the electron initial velocity spread are stopped by the effect of the permanent magnetic field. This influence is still valid even in the case of a wide gap between the 2 disks. Thus, at first approximation, one could solve the problem of the threshold determination by using the simple plane parallel model.

The next step will consist in taking into account the detailed secondary emission properties of the ferrite material by modeling them and then to insert them in higher level simulation tools to predict the Multipactor threshold in RF ferrite components taking into account the effect of the permanent magnetic field.

\section{References}

1. V. E. Semenov, N. A. Zharova, N. I. Zaitsev, A. K. Gvozdev, A. A. Sorokin, M. Lisak, J. Rasch, and J. Puech, "Reduction of the Multipactor Threshold Due to Electron Cyclotron Resonance", IEEE Transactions on Plasma Science, Vol. 40, No. 11, November 2012

2. D. Gonzalez-Iglesias, A. M. Perez, S. Anza, J. Vague, B. Gimeno, V. E. Boria, D. Raboso, C. Vicente, J. Gil, F. Caspers, and L. Conde, "Multipactor in a coaxial line with the presence of an axial DC magnetic field," IEEE Electron Device Lett., vol. 33, no. 5, pp. 727729,May 2012

3. M. Belhaj, J. Roupie, Th. Tondu, J-C. M. Vélez, J. Puech, N. Balcon, D. Payan, "Electron emission proprieties of conducting materials under low energy e-impact", 12th International Symposium on Materials in the Space Environment (ISMSE-12), 24-28 September 2012 ESA/ESTEC Noordwijk, The Netherlands

4. V. E. Semenov, N. A. Zharova, D. Anderson, M. Lisak, and J. Puech, "Simulations of multipactor in circular waveguides," Phys. Plasmas, vol. 17, no. 12, pp. 123 503-1-123 503-7, Dec. 2010..

5. J. R. M. Vaughan, "Secondary emission formulas," IEEE Trans. Electron Devices, vol. 40, no. 4, p. 830, Apr. 1993.

6. R.Udiljak, D. Anderson, M. Lisak, J. Puech, and V.E. Semenov, "Multipactor in a waveguide iris", IEEE Trans. Plasma Science 35, 388 (2007) 